\title{
Gouy phase anomaly in photonic nanojets
}

\author{
Myun-Sik Kim, ${ }^{1, a)}$ Toralf Scharf, ${ }^{1}$ Stefan Mühlig, ${ }^{2}$ Carsten Rockstuhl, ${ }^{2}$ and \\ Hans Peter Herzig ${ }^{1}$ \\ ${ }^{1}$ Optics and Photonics Technology Laboratory, Ecole Polytechnique Fédérale de Lausanne (EPFL), \\ Breguet 2, 2000 Neuchâtel, Switzerland \\ ${ }^{2}$ Institute of Condensed Matter Theory and Solid State Optics, Abbe Center of Photonics, \\ Friedrich-Schiller-Universität Jena, 07743 Jena, Germany
}

(Received 4 March 2011; accepted 14 April 2011; published online 13 May 2011)

\begin{abstract}
We investigate in real space amplitude and phase distributions of light in photonic nanojets emerging from micrometer-sized dielectric spheres with a high-resolution interference microscope. Strong localization of light and a Gouy phase anomaly are witnessed. We show that the phase advance of photonic nanojets significantly deviates from a plane wave due to the sudden transition from a converging to a diverging wave front. Understanding such phase anomalies and verifying the presence of photonic nanojets promises to pave the way to prospective applications that may exploit the ability to localize light in spatial domains smaller than the usual resolution limit. () 2011 American Institute of Physics. [doi:10.1063/1.3591175]
\end{abstract}

In miniaturized devices effects linked to the phase of light attracted an increasing importance. Predominantly because amplitude fields suffer from a finite spatial resolution and additional information from phase fields may lift that issue. ${ }^{1}$ Intrinsic to such high-resolution applications is the strong confinement that should lead to a phase anomaly. It is understood here as a deviation of the phase evolution of confined fields when compared to a plane wave. This so-called Gouy phase anomaly has been considered long time ago. Discovered by Gouy (Ref. 2) as early as 1890, this phenomena was studied in classical optics, for laser resonators and Gaussian beam propagation. ${ }^{3,4}$ The origin of the Gouy phase anomaly can be discussed from different theoretical perspectives. ${ }^{5-11}$ It is a phenomenon not just linked to Gaussian beams but it has been recently explored in the context of deviating wave fields. ${ }^{12}$ Along these lines of research we investigate here by experimental and numerical means the Gouy phase anomaly in spatially highly localized light fields.

There are several ways to create such highly confined light fields. The traditional approach is to strongly focus a Gaussian beam. This however imposes constraints since the Gaussian beam itself is only a solution to the paraxial wave equation and the onset of the vectorial nature of light makes it difficult to truly reach the resolution limit. An alternative approach to localize light even stronger relies on its coupling to the charge density oscillations in metals with plasmonic effects. ${ }^{13,14}$ Nonetheless, absorption and the associated strong spectral dispersion often constitutes an obstacle for prospective applications. Therefore, solutions to localize light relying exclusively on dielectrics are desirable. One is to use micrometer-sized dielectric spheres to generate highly localized light in the visible; called photonic nanojets. ${ }^{15}$ Such photonic nanojets generated a heated debate since their creation is considered as a scattering phenomenon and requires a rigorous treatment. ${ }^{16,17}$ Their fascination is attached to the promise to beat the diffraction limit at the expenses of being bound to the near- and intermediate-field region of the dielectric sphere. If photonic nanojets would be fully under-

\footnotetext{
${ }^{a)}$ Electronic mail: myunsik.kim@epfl.ch.
}

stood, their ability to steer the light in subwavelength domains could be exploited in many applications. ${ }^{18-21}$

We present here experimental results of fields emerging from photonic nanojets and discuss the Gouy phase anomaly in this context. All experimental results are supported by rigorous simulations. For our study we employ a highresolution interference microscope (HRIM) to measure amplitude and phase in the entire three-dimensional (3D) space. Details of the experimental setup are reported elsewhere. ${ }^{1,22}$ The underlying interferometer is based on a phase shifting algorithm and the Schwider-Hariharan method. ${ }^{23,24}$ All experimental and theoretical investigations were performed at a single wavelength of $642 \mathrm{~nm}$ (CrystaLaser: DL640-050-3). Microspheres are made of borosilicate glass and purchased from Duke Scientific Inc. Their refractive index is 1.55 at $642 \mathrm{~nm}$ and their diameter is $2 \mu \mathrm{m}$. Spheres are dispersedly deposited on the glass substrate and well isolated. A linearly polarized weakly focused Gaussian beam that can be safely approximated by a plane wave was used for the illumination. The photonic nanojets appear on the shadow-side surface of microspheres in air. The measured fields are low-pass filtered and the achievable spatial resolution for the amplitude fields is subject to the diffraction limit of the observing objective, a 100X/NA0.9 HC PL FLUOTAR from Leica Microsystems.

Figure 1 gives examples of intensity slices in longitudinal and transversal direction. The microsphere is indicated by a white circle. The incident light propagates along the positive $z$-axis and is polarized in $x$-direction. Measured fields which appear inside or spatially before the sphere do not correspond to fields in real space. They correspond to the back propagated fields which are transmitted in forward direction. The interferometer collects light only in transmission. Nevertheless, fields above the termination of the sphere correspond to those in the real space; with the only noticeable exception that their spatial frequency components are low-pass filtered by the optical system. The horizontal slice of the intensity through the plane of the largest spatial confinement is shown in Fig. 1(b).

The measured phase evolution of the fields is shown in Fig. 2. The phase is recorded as a differential phase along the propagation direction. Since the 3D measurements take sev- 

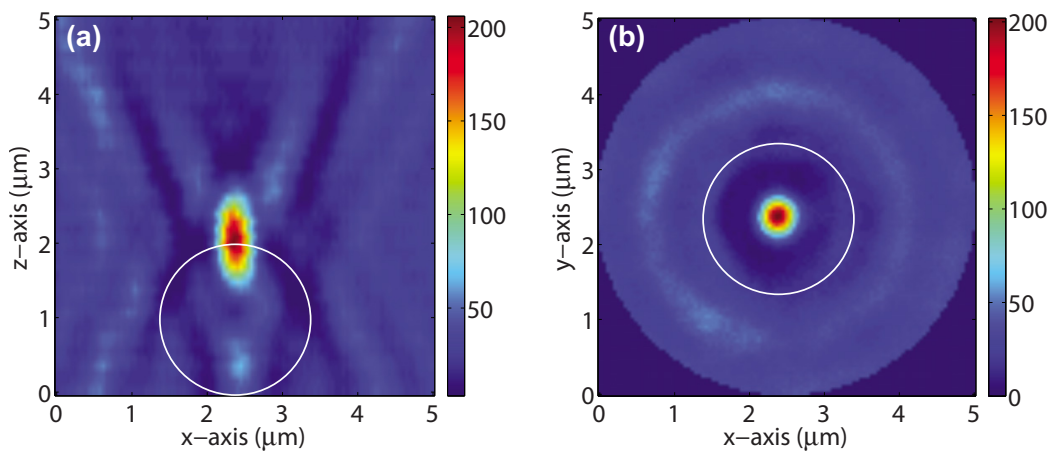

FIG. 1. (Color online) Measured intensity distributions of a photonic nanojet generated by a $2-\mu \mathrm{m}$ glass sphere: (a) the $x-z$ slice [at $y=2.4 \mu \mathrm{m}$ ] and (b) the $x-y$ slice [at $z=2 \mu \mathrm{m}$ ] through the center of the photonic nanojet. The white circle indicates the $2-\mu \mathrm{m}$ sphere.

eral minutes, there exists an inevitable phase drift. If the object however is small compared to the field of view, it can be assumed that a plane wave propagates far away from the object and the original differential phase can be corrected. We assume the differential phase of this plane wave to be constant and independent on the position. The differential phase after correction is shown in Fig. 2(a) and Fig. 2(b) shows a wrapped phase map to provide an impression of the pure phase evolution in space.

In order to provide a further understanding, we compare the experimental results to simulations. Mie theory for a single sphere was used to simulate the optical response of the glass sphere $(2 \mu \mathrm{m}$ diameter; $n=1.55) .{ }^{25}$ Assumptions on the illumination are the same as in the experiment. The substrate is neglected. The simulation of the optical imaging was performed using scalar propagation techniques in spatial frequency space. We calculate at first the $x$-component of the electric field $E_{\mathrm{x}}(x, y, z, \omega)$ (polarization of the incident field) with Mie theory in the $x$-y-plane at $z=10 \mu \mathrm{m}$. After Fourier transforming the field to the spatial frequency space $\left[\mathrm{E}_{\mathrm{x}}\left(k_{x}, k_{y}, z=10 \mu \mathrm{m}, \omega\right)\right]$, we suppress all contributions of spatial frequencies $\left(k_{x}\right.$ and $\left.k_{y}\right)$ whose absolute value is larger than the numerical aperture of the observing objective divided by the incident wavelength. This mimics the behavior of the objective in the experiments. Afterwards, this modified $x$-component of the electric field is propagated to the slice of interest in $z$-direction by means of free space propagation. So the field in the spatial frequency domain at every $z$ position reads as:

$$
E_{x}\left(k_{x}, k_{y}, z, \omega\right)=E_{x}\left(k_{x}, k_{y}, z=10 \mu m, \omega\right) e^{i k_{z} z} .
$$

The inverse Fourier transformation of this field into the real space gives the amplitude and phase. The differential and absolute phase maps constructed identical to the measured one are shown in Figs. 3(a) and 3(b). It has to be mentioned that this way of treating the optical image of the HRIM is only an approximation because we neglect all further optical elements. Anyhow, the good agreement between simulations and experiments seemingly justifies the approximations. Please note that only propagating components (and therefore tangential ones) of the electromagnetic field are detected by the HRIM and longitudinal ones (as they appear in the near field) cannot be observed. Therefore, the use of such scalar theory is adequate.

While the intensity in Fig. 1 don't reveal much information, the differential phase images in Figs. 2(a) and 3(a) show a phase variation with respect to a constant phase far away from the sphere, the Gouy phase anomaly. The peak intensity is found just behind the sphere at $z=2 \mu \mathrm{m}$. At the same position phase singularities appear left and right from the optical axis. Their lateral distance is measured to be $1+1$ $-0.1 \mu \mathrm{m}$ and agrees well with the distance found in the simulation of $0.90 \mu \mathrm{m}$. In passing we note that the field localization in real space is safely anticipated to be much stronger, since evanescent field components cannot be detected with the present setup. Moreover, the spatial distance between these phase singularities, which correspond to roots of the intensity, are much larger separated than the full-width at half-maximum (FWHM); a measure usually used to estimate the field localization in space. ${ }^{17}$ In measurements and simulations which consider the numerical aperture of the imaging objective this FWHM was $0.38 \mu \mathrm{m}$, in simulations which do not consider the imaging process but take the field directly behind the sphere it even was $0.25 \mu \mathrm{m}$; which corresponds to a localization of light in space in the order of $0.39 \lambda$.

The Gouy phase anomaly is a phase shift found along the $z$-axis (at $x=y=0 \mu \mathrm{m}$ ) and we plot the measured phase in Fig. 4. We explicitly show the differential phase where the advance from the plane wave is excluded, being fully in line with the definition of the Gouy phase anomaly. Experimental data from four individual measurements are shown. Since there is no analytical solution for the scattered hotspots, we extract as a theoretical reference results from the rigorous
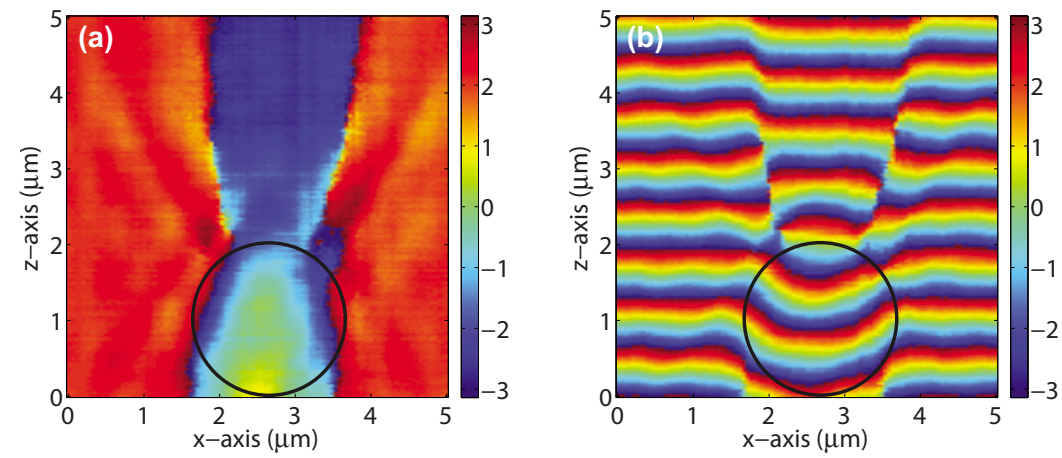

FIG. 2. (Color online) Measured phase distributions of a photonic nanojet spot in the axial direction. (a) Longitudinal map of differential phase with respect to the plane wave far away from the sphere. (b) The final wrapped phase that adds the phase advance of the plane wave to the differential phase. The black circle indicates the $2-\mu \mathrm{m}$ sphere. 

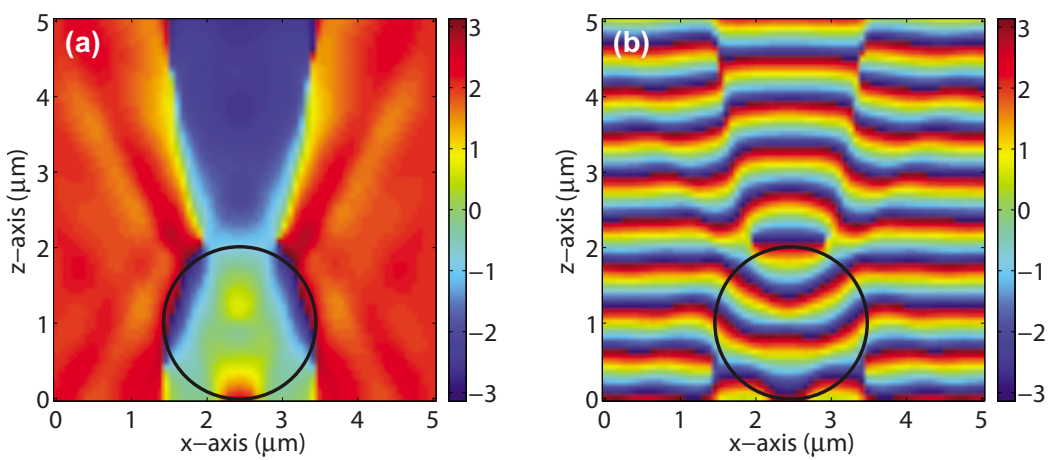

FIG. 3. (Color online) Corresponding simulation data to Figs. 2(a) and 2(b), which are calculated by Mie theory and scalar propagation technique: (a) differential phase map and (b) wrapped phase map. The black circle indicates the $2-\mu \mathrm{m}$ sphere.

simulations for $\mathrm{NA}=0.9$ [see Fig. 3(a) $]$ and $\mathrm{NA}=1$ and include the results as solid red and blue lines in Fig. 4, respectively. As long as almost all effective fields are captured by a $\mathrm{NA}=0.9$ acceptance angle, the $\mathrm{NA}=1$ case does not lead to different results.

Three different regimes can be distinguished along the $z$-axis. (1) Positions close to the substrate $[z=0-1 \mu \mathrm{m}]$ : The observation has aberrations and fields suffering multiple reflections between the substrate and the sphere causing experimental uncertainties. (2) Positions inside the sphere, at the intensity hotspot and behind the intensity hotspot $[z=1-3.5 \mu \mathrm{m}]$ : The high contrast of interference fringes and small amount of aberrations provide reliable results. Theory and measurement shows very good agreement in this region as expected. (3) Positions far away behind the hotspot $[z=3.5-5 \mu \mathrm{m}]$. As seen in Fig. 1(a), there is almost no intensity detectable and measured phases are subjected to noise. Nevertheless, the excellent agreement between simulation and experiment provides confidence that the results are sufficiently exact.

This longitudinal-differential phase measurement nicely demonstrates the Gouy phase anomaly at regions of the hotspot formation. Although an absolute discussion is difficult, it can be seen that in a very narrow spatial domain when compared to the wavelength, the phase advance of the field is strongly deviating. In order to confine the incident light fields in tiny spatial domains, the evolving wave fronts undergo a sudden transition from a spherical to a plane shape (converging) and from a plane to a spherical shape (diverging). This is comparable to a Gaussian beam which under-

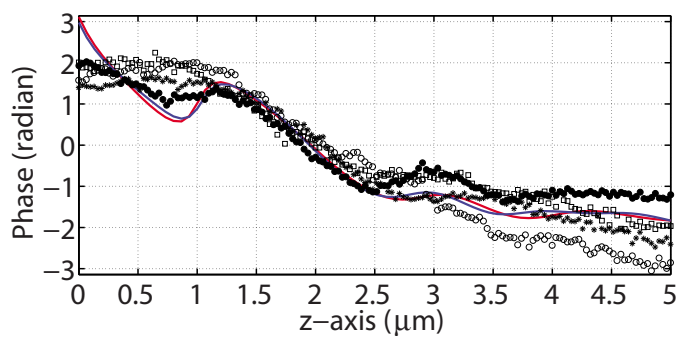

FIG. 4. (Color online) Profiles of differential phase in the axial direction $(z$-axis) along a central line of the photonic nanojets. The solid red and blue lines correspond to the rigorous simulations for $\mathrm{NA}=0.9$ and for $\mathrm{NA}=1$, respectively, and the various markers correspond to four individual measurements at a $2-\mu \mathrm{m}$ glass sphere. The filled circles correspond to the experimental results presented in Fig. 2(a). goes a focusing in its waist. The modifications to the wave fronts cause locally different propagation distances along the propagation direction and these are accumulated along the principal propagation direction. It results in a phase advance different to a plane wave; for which the Gouy phase is just a measure. This transition is strongest at the transition from a spherical to plane wave front and vice versa.

Also shown already in several ways for Gaussian beam propagation we demonstrate by experimental and theoretical means that a phase anomaly exists also in photonic nanojets. This supports the fact that the Gouy phase anomaly is a general phenomenon found for all kinds of a light confinement in space.

The research leading to these results has received funding from the European Community's Seventh Framework Programme FP7-ICT-2007-2 under Grant No. 224226.

${ }^{1}$ C. Rockstuhl, I. Märki, T. Scharf, M. Salt, H. P. Herzig, and R. Dändliker, Curr. Nanosci. 2, 337 (2006).

${ }^{2}$ L. G. Gouy, C. R. Hebd. Seances Acad. Sci. 110, 1251 (1890).

${ }^{3} \mathrm{M}$. Born and E. Wolf, Principles of Optics (Cambridge University Press, Cambridge, 1999).

${ }^{4}$ A. E. Siegman, Lasers (University Science Books, Stanford, 1986).

${ }^{5}$ P. Hariharan and P. A. Robinson, J. Mod. Opt. 43, 219 (1996).

${ }^{6}$ A. Rubinowicz, Phys. Rev. 54, 931 (1938).

${ }^{7}$ R. W. Boyd, J. Opt. Soc. Am. 70, 877 (1980).

${ }^{8}$ S. Feng and H. G. Winful, Opt. Lett. 26, 485 (2001).

${ }^{9}$ Y. Li, J. Opt. Soc. Am. A 2, 1677 (1985).

${ }^{10}$ Z. Derrar Kaddour, A. Taleb, K. Ait-Ameur, and G. Martel, Opt. Commun. 280, 256 (2007).

${ }^{11}$ J. Yang and H. G. Winful, Opt. Lett. 31, 104 (2006).

${ }^{12}$ T. D. Visser and E. Wolf, Opt. Commun. 283, 3371 (2010).

${ }^{13}$ C. Rockstuhl, C. R. Simovski, S. A. Tretyakov, and F. Lederer, Appl. Phys. Lett. 94, 113110 (2009).

${ }^{14}$ S. Mühlig, C. Rockstuhl, J. Pniewski, C. R. Simovski, S. A. Tretyakov, and F. Lederer, Phys. Rev. B 81, 075317 (2010).

${ }^{15}$ Z. Chen, A. Taflove, and V. Backman, Opt. Express 12, 1214 (2004).

${ }^{16}$ S. Lecler, Y. Takakura, and P. Meyrueis, Opt. Lett. 30, 2641 (2005).

${ }^{17}$ A. Heifetz, S.-C. Kong, A. V. Sahakian, A. Taflove, and V. Backman, J. Comput. Theor. Nanosci. 6, 1979 (2009).

${ }^{18}$ X. Li, Z. Chen, A. Taflove, and V. Backman, Opt. Express 13, 526 (2005).

${ }^{19}$ S.-C. Kong, A. Sahakian, A. Taflove, and V. Backman, Opt. Express 16, 13713 (2008).

${ }^{20}$ E. Mcleod and C. B. Arnold, Nat. Nanotechnol. 3, 413 (2008).

${ }^{21}$ Z. Wang, W. Guo, L. Li, B. Luk'yanchuk, A. Khan, Z. Liu, Z. Chen, and M. Hong, Nature Commun. 2, 218 (2011).

${ }^{22}$ M.-S. Kim, T. Scharf, and H. P. Herzig, Opt. Express 18, 14319 (2010).

${ }^{23}$ J. Schwider, R. Burow, K.-E. Elssner, J. Grzanna, R. Spolaczyk, and K. Merkel, Appl. Opt. 22, 3421 (1983).

${ }^{24}$ P. Hariharan, B. F. Oreb, and T. Eiju, Appl. Opt. 26, 2504 (1987).

${ }^{25}$ C. F. Bohren and D. R. Huffman, Absorption and Scattering of Light by Small Particles (Wiley, New York, 1983). 